\title{
Comparison of chemical pregnancy rates according to the anesthetic method during ultrasound-guided transvaginal oocyte retrieval for in vitro fertilization: a retrospective study
}

Received May 31, 2019

Revised July 8, 2019

Accepted July 19, 2019

\section{Corresponding author}

Yu Yil Kim, M.D.

Department of Anesthesiology and Pain Medicine, Presbyterian Medical Center, 365 Seowon-ro, Wansan-gu, Jeonju 54987, Korea

Tel: 82-63-230-1594

Fax: 82-63-230-8919

E-mail: gangoaogi@naver.com

\section{ORCID}

https://orcid.org/0000-0003-3455-9251

\section{Hyun Joo Heo, Yu Yil Kim, Ji Hye Lee, Han Gyeol Lee, Seung Min Baek, and Ki Man Kim}

Department of Anesthesiology and Pain Medicine, Presbyterian Medical Center, Jeonju, Korea

\begin{abstract}
Background: Oocyte retrieval is the most important procedure in in vitro fertilization (IVF). Various anesthetic methods are used to control a patient's anxiety and pain during IVF; however, there are no recommended anesthetic methods at present. In this study, we retrospectively investigated chemical pregnancy rates according to the anesthetic method used for oocyte retrieval.
\end{abstract}

Methods: We reviewed records of patients who underwent oocyte retrieval between January 1, 2012 and December 31, 2017. Patients were divided into the spinal anesthesia (SA) and monitored anesthesia care (MAC) groups. The primary outcome was chemical pregnancy rate after IVF.

Results: The study included 95 patients. SA was administered in 77 (81\%) and MAC in 18 (19\%). The overall chemical pregnancy rate was 32.6\% (31/95). According to the anesthetic method, the pregnancy rate was 32.5\% (25/77) in the SA group and 33.3\% $(6 / 18)$ in the MAC group. There was no statistical difference in the pregnancy rate between the groups $(P=0.575)$. The procedural time was significantly shorter in the SA group than in the MAC group $(P<0.001)$.

Conclusions: Chemical pregnancy rates were not significantly different between the SA and MAC groups. However, the procedure duration was shorter in the SA group than in the MAC group.

Keywords: Anesthesia; In vitro fertilization; Oocyte retrieval.

\section{INTRODUCTION}

In vitro fertilization (IVF) is the most commonly used assisted reproductive technique worldwide. Of the several processes involved in IVF, one of the most important process is transvaginal oocyte retrieval (TVOR), in which oocytes are obtained from the ovarian follicles via ultrasound-guided needle aspiration through the vaginal wall. Although TVOR is relatively simple and less invasive, the patient can experience anxiety and pain during the procedure. Therefore, various drugs and anesthetic methods, such as general anesthesia, monitored anesthesia care (MAC) or conscious sedation, and regional anesthesia, are used to manage the patient's anxiety and pain, as well as to ensure a stable procedural environment.

There is no established anesthetic method for TVOR, although there have been many studies on the effects of anesthetic methods and drugs used during TVOR on the outcome

This is an Open Access article distributed under the terms of the Creative Commons Attribution Non-Commercial License (http://creativecommons.org/licenses/by-nc/4.0) which permits unrestricted non-commercial use, distribution, and reproduction in any medium, provided the original work is properly cited. 
of IVF [1]. Therefore, the purpose of this study was to retrospectively investigate the effects of anesthetic methods on the chemical pregnancy rate in patients who underwent IVF between January 1, 2012 and December 31, 2018.

\section{MATERIALS AND METHODS}

This study was approved by the Institutional Review Board of our hospital (no. 2019-04-011).

We included 97 patients who underwent IVF after TVOR between January 1, 2012 and December 31, 2018. Among them, two patients were excluded: one patient who rejected fertilized embryo transfer and another in whom oocyte retrieval failed.

Patients' medical records were reviewed for demographic information, anesthetic method used for oocyte retrieval, and chemical pregnancy rate.

All patients underwent ultrasound-guided TVOR after ovarian hyperstimulation conducted by the same surgeon, followed by fertilization, embryo culture, and intrauterine transplantation at the same institution. The pregnancy outcome was confirmed approximately 10 days after embryo transfer through serum $\beta$-human chorionic gonadotropin evaluation in only the first IVF trial with fresh oocytes. Two anesthetic methods were used during TVOR: MAC and spinal anesthesia (SA). The first-choice anesthetic method was SA; however, according to the patient's choice and when the patient had conditions precluding the use of SA, such as allergy to local anesthetics, MAC was used. No sedation was used at the time of SA, whereas MAC was provided with a propofol target-controlled pump infusion system.

Statistical analysis was performed using SPSS Statistics soft-

Table 1. Characteristics of Patients

\begin{tabular}{lccc}
\hline \multicolumn{1}{c}{ Characteristic } & $\mathrm{SA}(\mathrm{n}=77)$ & $\mathrm{MAC}(\mathrm{n}=18)$ & P value \\
\hline Age $(\mathrm{yr})$ & $34.0 \pm 5.0$ & $35.8 \pm 5.7$ & 0.195 \\
Weight $(\mathrm{kg})$ & $61.7 \pm 13.2$ & $56.9 \pm 6.2$ & 0.025 \\
Height $(\mathrm{cm})$ & $158.8 \pm 6.0$ & $154.2 \pm 5.4$ & 0.004 \\
$\mathrm{BMI}\left(\mathrm{kg} / \mathrm{m}^{2}\right)$ & $24.0 \pm 3.0$ & $24.4 \pm 4.8$ & 0.623 \\
Procedure duration (min) & $27.7 \pm 9.4$ & $38.6 \pm 9.2$ & 0.000 \\
SA level (thoracic) & $8.5 \pm 1.7$ & - & - \\
Hyperbaric bupivacaine $(\mathrm{mg})$ & $10.9 \pm 1.1$ & - & - \\
Propofol dose $(\mathrm{mg})$ & - & $303.9 \pm 48.2$ & - \\
\hline
\end{tabular}

Values are presented as mean \pm SD. SA: spinal anesthesia, MAC: monitored anesthesia care, BMl: body mass index. ware (version 23, IBM SPSS Inc., USA), and the demographic information of patients was expressed as mean \pm standard deviation. The pregnancy outcome according to anesthetic method was analyzed using Fisher's exact test. P values $<0.05$ were considered statistically significant.

\section{RESULTS}

During TVOR, SA was used in 77 patients (81\%) and MAC with continuous intravenous drug infusion was used in 18 (19\%). The two groups showed differences in height and body weight; however, body mass index was not significantly different. The procedure duration was significantly shorter with SA than with MAC (Table 1). There was no anesthetic failure in the SA group. In two patients in the SA group, the mean arterial pressure was $<60 \mathrm{mmHg}$ and $5 \mathrm{mg}$ ephedrine was administered. No other complications were observed. The overall chemical pregnancy rate after IVF was $32.6 \%$ (31/95), and there was no difference in the pregnancy rates between the two groups (32.5\% [25/77] in the SA group and 33.3\% [6/18] in the MAC group; $\mathrm{P}=0.575$ ). In the case of oocyte retrieval with MAC, patient movements during the procedure were observed in three patients; however, no procedure-related complications occurred. Neither respiratory depression nor hemodynamic instability was observed during MAC.

\section{DISCUSSION}

The IVF procedure consists of ovarian hyperstimulation, oocyte retrieval, fertilization, embryo culture, and embryo transfer, among which oocyte retrieval is the most invasive. In the past, oocyte retrieval was performed using laparoscopy; however, laparoscopy has been replaced by ultrasound-guided TVOR. Although TVOR is less invasive and easier to perform than the laparoscopic approach, patients still feel anxious about the procedure. Penetration of the vaginal wall and ovarian capsule with a needle during TVOR causes discomfort and pain, which are increased with repeated attempts in cases in which the involvement of many follicles is required for adequate oocyte retrieval [2]. Such discomfort and pain consequently cause patients to make unexpected movements during the procedure, which can lead to procedural complications such as bleeding and tissue damage. Therefore, it is necessary to use appropriate methods to control anxiety, dis- 
comfort, and pain in patients, as well as to provide surgeons with a stable environment for the procedure.

An ideal anesthetic method for oocyte retrieval should (i) have fast action and short recovery time, (ii) provide adequate pain control and sedation with an agent that does not accumulate in the follicular fluid and does not inhibit the outcome of IVF by affecting the oocytes and embryo, and (iii) have no effect on IVF outcomes. General anesthesia, MAC or conscious sedation, and regional or local anesthesia are used for the safe and effective treatment of patients during TVOR. Although many studies have investigated the effect of the anesthesia used during TVOR on IVF outcomes, no definite results have been reported to date [1].

General anesthesia is now used restrictively because the laparoscopic method of oocyte retrieval has been replaced by the relatively simpler and less invasive TVOR. Further, reports have demonstrated inferior outcomes of IVF performed under general anesthesia to those of IVF performed using MAC and regional (spinal, epidural, and paracervical block) anesthesia. Azumude et al. [3] reported that SA increased the success rate of IVF and that a relatively lower volume of drug was used for SA than for general anesthesia. Aghaamoo et al. [4] recommended SA over general anesthesia for oocyte retrieval. In addition, Wilhelm et al. [5] reported a higher pregnancy rate in patients who received MAC than in those who received general anesthesia. However, Hammadeh at al. [6] reported that general anesthesia led to a higher oocyte retrieval rate than sedation by eliminating the anxiety of patients, thereby providing a more stable and comfortable procedural environment for surgeons. In a study comparing SA with intravenous sedation, pregnancy rates were similar between the two methods, although the procedure duration was longer and many complications, such as nausea, occurred in patients who received intravenous sedation [7].

Anesthetic drugs used in TVOR should have fast action, short recovery time, and no detrimental effect on the oocytes and embryo. Midazolam can cause anxiolysis and anterograde amnesia, and is often used as a premedication or sedation drug in simple procedures; however, few studies on its effects on IVF have been conducted. Propofol is the most commonly used intravenous anesthetic for rapid effect and recovery and is commonly used in MAC or conscious sedation during IVF. Although propofol can accumulate in the follicular fluid [8,9], results of studies that examined the effects of propofol on IVF outcomes have been contradictory [10-12]. Previous studies suggested that propofol is a relatively safe drug but should be used with caution [9,11-13]. Opioids (commonly fentanyl, alfentanil, and remifentanil) can be used to control TVOR-associated pain. Compared with fentanyl, remifentanil leads to a higher IVF pregnancy rate [14]. Meanwhile, alfentanil must be cautiously used, as it is associated with a higher incidence of respiratory depression than is fentanyl [15].

In this study, similar chemical pregnancy rates were observed in the MAC and SA groups. However, the procedure duration was longer with MAC, which may be because SA provides a more comfortable procedural environment than MAC (during which patients may have unintended movements). Conscious sedation with sedatives and opioids or MAC involves continuous infusion of drugs or additional drug administration after bolus, if necessary. The use of target-controlled infusion and anesthetic depth monitoring may help reduce the volume of drugs used and allow faster recovery while minimizing the effects on the oocytes and embryo. Moreover, compared with conscious sedation, MAC allows anesthesiologists to control the sedation level, pain, and changes in the state of the patient during the procedure, thereby ensuring the safety of patients and helping surgeons perform the procedure stably and effectively.

This study is limited by its retrospective design and the disproportionate number of patients in the two groups. Nevertheless, it demonstrates that SA is a useful anesthetic method in TVOR during IVF and allows a shorter TVOR procedure duration than MAC. However, considering that TVOR is a day procedure, MAC may also be a good choice with the careful use of anesthetic drugs.

\section{CONFLICTS OF INTEREST}

No potential conflict of interest relevant to this article was reported.

\section{ORCID}

Hyun Joo Heo, https://orcid.org/0000-0003-2507-6629

Ji Hye Lee, https://orcid.org/0000-0003-3269-3844

Han Gyeol Lee, https://orcid.org/0000-0001-7835-0172

Seung Min Baek, https://orcid.org/0000-0003-0290-0208 
Ki Man Kim, https://orcid.org/0000-0002-4257-9167

\section{REFERENCES}

1. Matsota P, Kaminioti E, Kostopanagiotou G. Anesthesia related toxic effects on in vitro fertilization outcome: burden of proof. Biomed Res Int 2015; 2015: 475362.

2. Gejervall AL, Stener-Victorin E, Möller A, Janson PO, Werner C, Bergh C. Electro-acupuncture versus conventional analgesia: a comparison of pain levels during oocyte aspiration and patients' experiences of well-being after surgery. Hum Reprod 2005; 20: 728-35.

3. Azmude A, Agha'amou S, Yousefshahi F, Berjis K, Mirmohammad'khani M, Sadaat'ahmadi F, et al. Pregnancy outcome using general anesthesia versus spinal anesthesia for in vitro fertilization. Anesth Pain Med 2013; 3: 239-42.

4. Aghaamoo S, Azmoodeh A, Yousefshahi F, Berjis K, Ahmady F, Qods K, et al. Does spinal analgesia have advantage over general anesthesia for achieving success in in-vitro fertilization? Oman Med J 2014; 29: 97-101.

5. Wilhelm W, Hammadeh ME, White PF, Georg T, Fleser R, Biedler A. General anesthesia versus monitored anesthesia care with remifentanil for assisted reproductive technologies: effect on pregnancy rate. J Clin Anesth 2002; 14: 1-5.

6. Hammadeh ME, Wilhelm W, Huppert A, Rosenbaum P, Schmidt W. Effects of general anaesthesia vs. sedation on fertilization, cleavage and pregnancy rates in an IVF program. Arch Gynecol Obstet 1999; 263: 56-9.

7. Viscomi CM, Hill K, Johnson J, Sites C. Spinal anesthesia versus intravenous sedation for transvaginal oocyte retrieval: reproductive outcome, side-effects and recovery profiles. Int J Obstet Anesth 1997; 6: 49-51.

8. Christiaens F, Janssenswillen C, Verborgh C, Moerman I, Dev- roey P, Van Steirteghem A, et al. Propofol concentrations in follicular fluid during general anaesthesia for transvaginal oocyte retrieval. Hum Reprod 1999; 14: 345-8.

9. Coetsier T, Dhont M, De Sutter P, Merchiers E, Versichelen L, Rosseel MT. Propofol anaesthesia for ultrasound guided oocyte retrieval: accumulation of the anaesthetic agent in follicular fluid. Hum Reprod 1992; 7 : 1422-4.

10. Piroli A, Marci R, Marinangeli F, Paladini A, Di Emidio G, Giovanni Artini P, et al. Comparison of different anaesthetic methodologies for sedation during in vitro fertilization procedures: effects on patient physiology and oocyte competence. Gynecol Endocrinol 2012; 28: 796-9.

11. Ben-Shlomo I, Moskovich R, Golan J, Eyali V, Tabak A, Shalev E. The effect of propofol anaesthesia on oocyte fertilization and early embryo quality. Hum Reprod 2000; 15: 2197-9.

12. Matsota P, Sidiropoulou T, Batistaki C, Giannaris D, Pandazi A, Krepi $\mathrm{H}$, et al. Analgesia with remifentanil versus anesthesia with propofol-alfentanil for transvaginal oocyte retrieval: a randomized trial on their impact on in vitro fertilization outcome. Middle East J Anaesthesiol 2012; 21: 685-92.

13. Tewari S, Bhadoria P, Wadhawan S, Prasad S, Kohli A. Entropy vs standard clinical monitoring using total intravenous anesthesia during transvaginal oocyte retrieval in patients for in vitro fertilization. J Clin Anesth 2016; 34: 105-12.

14. Jarahzadeh MH, Davar R, Hajiesmaeili MR, Entezari A, Musavi F. Remifentanil versus fentanyl for assisted reproductive technologies: effect on hemodynamic recovery from anesthesia and outcome of ART cycles. Int J Fertil Steril 2011; 5: 86-9.

15. Kang YI, Bang EC, Lee HS, Cho KS, Kim SY, Sul JH, et al. The comparison of intravenous anesthesia with fentanyl versus alfentanill during oocyte retrieval for in vitro fertilization. Korean J Anesthesiol 2008; 55: 543-8. 